\title{
基于稀土阳离子和多酸阴离子的系列纯无机离子液体的合成及性质
}

\author{
王赫男张安歌张仲田洪瑞岳倩赵雪鹿颖刘术侠* \\ (东北师范大学 多酸与网格材料化学教育部重点实验室 长春 130024)
}

\begin{abstract}
摘要 本工作报道了系列钛取代 Keggin 型钨磷酸稀土盐 $\mathrm{KLnH}_{3} \mathrm{PTi}_{2} \mathrm{~W}_{10} \mathrm{O}_{40} \bullet x \mathrm{H}_{2} \mathrm{O}[\mathrm{Ln}=\mathrm{La}(\mathbf{1}), \mathrm{Ce}(\mathbf{2}), \mathrm{Pr}(\mathbf{3}), \mathrm{Nd}(\mathbf{4}), \mathrm{Sm}$ (5), $\mathrm{Eu}(6), \mathrm{Er}(7), \mathrm{Yb}(8)$ 的合成和性质. 这些无机离子型化合物在 $4 \sim 65{ }^{\circ} \mathrm{C}$ 范围表现为流动性很好的液态, 呈现离子 液体行为. 微量的水是该系列离子液体组成中不可缺少的组分, 完全失去水后变为无定型的透明固体. 该系列离子液 体在室温时具有良好的导电性, 电导率均高于 $10 \mathrm{mS} \cdot \mathrm{cm}^{-1}$, 而且其电导率随温度发生明显的变化. 当从室温升高到 $65{ }^{\circ} \mathrm{C}$ 时, 含 $\mathrm{Ce}$ 的离子液体 2 电导率从 $13.3 \mathrm{mS} \cdot \mathrm{cm}^{-1}$ 逐渐增大至 $22.6 \mathrm{mS} \cdot \mathrm{cm}^{-1}$, 但从 $65{ }^{\circ} \mathrm{C}$ 升温至 $90{ }^{\circ} \mathrm{C}$ 时, 电导率明 显降低. 研究还发现, 在室温环境下该系列离子液体与水均不互溶呈两相, 加热后混溶为均一相, 表现出上临界溶解 温度(UCST)相行为. 据我们所知, 这种纯无机离子液体是非常少见的.
\end{abstract}

关键词 钛取代 Keggin 型铇磷酸; 稀土; 无机离子液体; 电导率; 上临界溶解温度(UCST)相行为

\section{Synthesis and Properties of a Series of Pure Inorganic Ionic Liquids Based on Rare Earth Cations and Polyoxometalates}

\author{
He-Nan Wang An-Ge Zhang Zhong Zhang Hong-Rui Tian Qian Yue Xue Zhao \\ Ying Lu Shu-Xia Liu* \\ (Key Laboratory of Polyoxometalate and Reticular Material Chemistry of Ministry of Education, \\ Northeast Normal University, Changchun 130024, China)
}

\begin{abstract}
Ionic liquids (ILs) usually refer to ionic compounds that are liquid at a temperature of $100{ }^{\circ} \mathrm{C}$ and below. As salts, ionic liquids are composed of ions. So by selecting the corresponding cations, anions or ion combinations, they can be designed for specific applications. Various new types of ionic liquids have been prepared and discovered. Polyoxometalates (POMs) are a kind of metal-oxygen cluster compounds with rich composition and diverse structure. Compared with other common inorganic anions (such as $\mathrm{PO}_{4}{ }^{3-}, \mathrm{SO}_{4}{ }^{2-}, \mathrm{CO}_{3}{ }^{2-}$, etc.), polyoxometalate anions have the characteristics of large ion radius and low charge density, which are a good anion choice for constructing ionic liquids. Most of the currently reported polyoxometalate ionic liquids are composed of organic cations and polyoxometalate anions, while pure inorganic ionic liquids composed of inorganic cations and polyoxometalate anions are rarely reported. In this work, we report a series of lanthanide- containing compounds based on a Ti-substituted Keggin-type polyoxometalate prepared by ion exchange, $\mathrm{KLnH}_{3} \mathrm{PTi}_{2} \mathrm{~W}_{10} \mathrm{O}_{40} \bullet x \mathrm{H}_{2} \mathrm{O}$ [Ln= $\mathrm{La}(\mathbf{1}), \mathrm{Ce}(\mathbf{2}), \mathrm{Pr}(\mathbf{3}), \mathrm{Nd}(\mathbf{4}), \mathrm{Sm}(\mathbf{5}), \mathrm{Eu}(\mathbf{6}), \mathrm{Er}(7), \mathrm{Yb}(\mathbf{8})$ ]. They were characterized by inductively coupled plasma emission spectrometry (ICP), thermal gravimetric analysis (TG), Fourier transform infrared (FT-IR) spectroscopy, powder X-ray diffraction (PXRD). Surprisingly, these inorganic ionic compounds behave as liquid with good fluidity in the temperature range of $4 \sim 65{ }^{\circ} \mathrm{C}$, and their conductivity are all higher than $10 \mathrm{mS} \bullet \mathrm{cm}^{-1}$, showing ionic liquid behavior. Water is an indispensable component in their composition. After losing water, they become transparent and colored solids with amorphous structure. The study found that the conductivity of these ionic liquids change significantly with temperature. For example, when the temperature rises from room temperature to $65{ }^{\circ} \mathrm{C}$, the conductivity of Ce-containing ionic liquid gradually increases from $13.3 \mathrm{mS} \cdot \mathrm{cm}^{-1}$ to $22.6 \mathrm{mS} \cdot \mathrm{cm}^{-1}$. When the temperature rises from $65{ }^{\circ} \mathrm{C}$ to $90{ }^{\circ} \mathrm{C}$, the conductivity drops significantly, as low as $1.22 \mathrm{mS} \cdot \mathrm{cm}^{-1}$ at $90{ }^{\circ} \mathrm{C}$. As far as we know, this kind of pure inorganic ionic liquid is very rare. The synthesis method of series ionic liquids is simple, easy to operate, and environmentally friendly. These properties make the series of ionic liquids have good machinability and potential applications in the fields of catalysis and phase separation.
\end{abstract}

Keywords Ti-substituted Keggin-type polyoxometalate; rare earth; inorganic ionic liquids; conductivity; upper critical solution temperature (UCST)

\section{1 引言}

经典的离子液体 (ionic liquids, ILs) 是指在温度
$100{ }^{\circ} \mathrm{C}$ 及以下仍呈液态的离子型化合物 ${ }^{[1-2]}$. 与传统的 分子型溶剂相比，离子液体具有高的离子导电性、宽的

*E-mail: liusx@nenu.edu.cn

Received April 2, 2021; published April 24, 2021.

Supporting information for this article is available free of charge via the Internet at http://sioc-journal.cn.

Project supported by the National Natural Science Foundation of China (Nos. 21872021, 21671033, 22071019).

项目受国家自然科学基金(Nos. 21872021, 21671033, 22071019)资助. 
电化学窗口、低的蒸汽压以及不易然等特性 ${ }^{[3]}$. 近些年, 随着各种新型离子液体被制备和发现，离子液体的应用

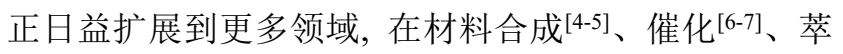

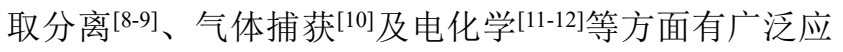
用.

多金属氧酸盐(polyoxometalates, POMs)是一类组成 丰富、结构多样的金属-氧簇合物, 具有优异的物理化学 性质, 被广泛应用于催化、材料、生物医药等领域 ${ }^{[13-15]}$. 与其它常见的无机阴离子(如 $\mathrm{PO}_{4}{ }^{3-}, \mathrm{SO}_{4}{ }^{2-}, \mathrm{CO}_{3}{ }^{2-}$ 等)相 比, 多金属氧族阴离子具有离子半径大、电荷密度低的 特点, 是构筑离子液体很好的阴离子选择. 目前, 已报 道很多用咪唑、吡啶、季胺类阳离子与多金属氧酸盐阴 离子构筑的离子液体. 例如, Leng 等 ${ }^{[16]}$ 设计合成系列多 酸基离子液体 $[\mathrm{MIMPS}]_{3} \mathrm{PW}_{12} \mathrm{O}_{40},[\mathrm{PyPS}]_{3} \mathrm{PW}_{12} \mathrm{O}_{40}$ 和 [TEAPS $]_{3}-\mathrm{PW}_{12} \mathrm{O}_{40}$, 并研究它们在酯化反应中的催化 行为. Herrmann 等 ${ }^{[17]}$ 报道了过渡金属取代的 Keggin 多 酸阴离子 $\left[\mathrm{SiW}_{11} \mathrm{O}_{39} \mathrm{TM}\left(\mathrm{H}_{2} \mathrm{O}\right)\right]^{n-}\left(\mathrm{TM}=\mathrm{Cu}^{\mathrm{II}}, \mathrm{Fe}^{\mathrm{III}}\right)$ 和季烷 基铵阳离子 $\left(\mathrm{C}_{n} \mathrm{H}_{2 n+1}\right)_{4} \mathrm{~N}^{+}(n=7 \sim 8)$ 形成的室温多酸基 离子液体, 用于自修复金属腐蚀防护涂层. Qiao 等 ${ }^{[18]}$ 用 质子化烷基咪唑与过氧铇酸盐制备了具有离子液体行 为的复合物 $[\mathrm{HDIm}]_{2}\left[\left\{\mathrm{WO}\left(\mathrm{O}_{2}\right)_{2}\right\}_{2}(\mu-\mathrm{O})\right]$, 其对环辛烯的 环氧化体现出非常有效的催化作用.

目前报道的多酸基离子液体大多是由有机阳离子 与多酸阴离子构成. 据我们所知, 由无机阳离子与多酸 阴离子构成的纯无机离子液体仅有一例 ${ }^{[19]}$. 在本工作 中, 我们以镧系金属盐和钛取代的 Keggin 型多金属氧 酸盐 $\left(\mathrm{K}_{7} \mathrm{PTi}_{2} \mathrm{~W}_{10} \mathrm{O}_{40}\right)$ 为原料, 合成了系列新的基于多金 属氧酸盐的无机离子液体 $\mathrm{KLnH}_{3} \mathrm{PTi}_{2} \mathrm{~W}_{10} \mathrm{O}_{40} \bullet x \mathrm{H}_{2} \mathrm{O}[\mathrm{Ln}$ $=\mathrm{La}(\mathbf{1}), \mathrm{Ce}(\mathbf{2}), \operatorname{Pr}(\mathbf{3}), \mathrm{Nd}(\mathbf{4}), \operatorname{Sm}(\mathbf{5}), \operatorname{Eu}(\mathbf{6}), \operatorname{Er}(\mathbf{7}), \mathrm{Yb}(\mathbf{8})]$, 通过电感耦合等离子体原子发射光谱(ICP)、热重(TG)、 红外光谱(IR)、粉末 X-射线衍射(PXRD)、循环伏安 $(\mathrm{CV})$ 等进行表征确定其组成和性质. 研究发现, 这些离子液 体与水混合表现出上临界溶解温度(UCST)相行为. 该 系列离子液体组成中水是其不可缺少的组分, 失去水后 转化为固态, 但组成结构不发生变化, 失水后的固态物 质加入适量水可以重新得到流动性很好的液体.

\section{2 结果与讨论}

\section{1 合成方法}

$\mathrm{K}_{7} \mathrm{PTi}_{2} \mathrm{~W}_{10} \mathrm{O}_{40}$ 按照文献 ${ }^{[20]}$ 方法合成, 并通过红外光 谱和粉末 X-射线衍射分析进行验证, 其衍射峰与单晶 模拟的 PXRD 谱图比对良好(如图 3a, 3b).

将稀土硝酸盐乙醇溶液逐滴加入 $\mathrm{K}_{7} \mathrm{PTi}_{2} \mathrm{~W}_{10} \mathrm{O}_{40}$ 水 溶液中 $(\mathrm{pH}$ 约 7.5), 其物质的量比为 $4: 3$, 回流反应 20 $\min$ 后, 在室温下静置约 $1 \mathrm{~h}$ 至析出油状物, 分离出油状 物即为系列离子液体(离子液体 $\mathbf{1} \sim 8$ ), 如图 1a 所示. 经 元素分析(表 S1)和热重分析(图 S1)等确定其化学式为 $\mathrm{KLnH}_{3} \mathrm{PTi}_{2} \mathrm{~W}_{10} \mathrm{O}_{40} \bullet x \mathrm{H}_{2} \mathrm{O}[\mathrm{Ln}=\mathrm{La}(\mathbf{1}), \mathrm{Ce}(\mathbf{2}), \mathrm{Pr}(\mathbf{3}), \mathrm{Nd}$
(4), $\mathrm{Sm}(5), \mathrm{Eu}(6), \operatorname{Er}(7), \mathrm{Yb}(\mathbf{8})]$.

实验中取少量系列离子液体放置在 $50{ }^{\circ} \mathrm{C}$ 烘箱中 48 $\mathrm{h}$, 得到透明固体(固体 $\mathbf{1 s} \sim \mathbf{8 s}$ ), 其颜色由于稀土离子不 同而有所区别，如图 1b. 这些固体分别加入适量水可重 新获得油状物(油状物 10 80).

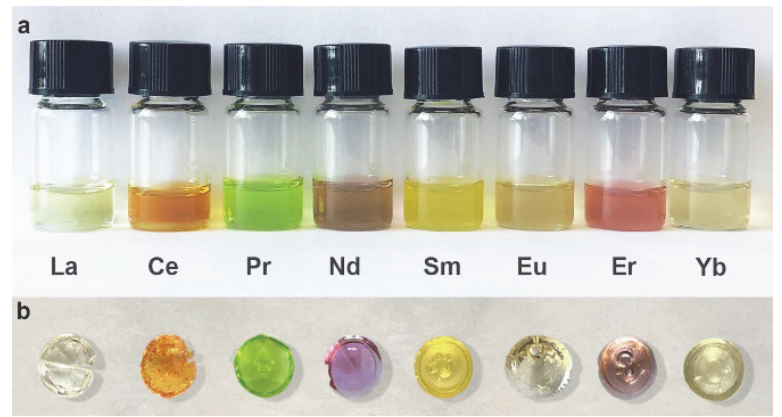

图 1 (a)系列离子液体 $(\mathbf{1} \sim 8)$; (b)系列离子液体 $50{ }^{\circ} \mathrm{C}$ 干燥 $48 \mathrm{~h}$ 所得 固体 $(\mathbf{1 s} \sim \mathbf{8 s})$

Figure 1 (a) Serious of ionic liquids $(\mathbf{1} \sim \mathbf{8})$; (b) The solids $(\mathbf{1 s} \sim \mathbf{8 s})$ obtained by drying the series of ionic liquids at $50{ }^{\circ} \mathrm{C}$ for $48 \mathrm{~h}$

在合成 $\mathrm{KLnH}_{3} \mathrm{PTi}_{2} \mathrm{~W}_{10} \mathrm{O}_{40} \bullet x \mathrm{H}_{2} \mathrm{O}[\mathrm{Ln}=\mathrm{La}(\mathbf{1}), \mathrm{Ce}(\mathbf{2})$, $\operatorname{Pr}(3), \mathrm{Nd}(4), \mathrm{Sm}(5), \mathrm{Eu}(6), \operatorname{Er}(7), \mathrm{Yb}(8)]$ 时, 如果直接 将稀土硝酸盐与 $\mathrm{K}_{7} \mathrm{PTi}_{2} \mathrm{~W}_{10} \mathrm{O}_{40}$ 的水溶液混合、搅拌反应, 会发现有大量沉淀生成. 元素分析该沉淀中各元素物质 的量比为 $n(\mathrm{~K}): n(\mathrm{Ln}): n(\mathrm{P}): n(\mathrm{Ti}): n(\mathrm{~W})=1: 2: 1:$ $2: 10$, 与化学式 $\mathrm{KLn}_{2} \mathrm{PTi}_{2} \mathrm{~W}_{10} \mathrm{O}_{40}$ 一致, 这也符合正盐 的溶解度一般低于酸式盐 $\left(\mathrm{KLnH}_{3} \mathrm{PTi}_{2} \mathrm{~W}_{10} \mathrm{O}_{40}\right)$ 的规律. 如果我们控制稀土硝酸盐的加入量, 在观察到沉淀生成 就立刻停止加入并过滤, 随后向滤液中加入乙醇并在冰 箱 $\left(4{ }^{\circ} \mathrm{C}\right)$ 中放置过夜, 仅得到少量的油状物, 产率极低. 但如果将稀土硝酸盐溶解在少量乙醇中, 然后滴加到 $\mathrm{K}_{7} \mathrm{PTi}_{2}-\mathrm{W}_{10} \mathrm{O}_{40}$ 水溶液中, 开始看到有絮状物产生, 之 后在室温下静置就可得到大量油状物. 据此我们推测, 醇的加入可能与稀土离子形成相对稳定的加合物，尽管 将其加入到偏中性的 $\mathrm{K}_{7} \mathrm{PTi}_{2} \mathrm{~W}_{10} \mathrm{O}_{40}$ 水溶液中, 稀土离子 不至于发生水解, 而是与多酸阴离子形成酸式盐. 元素 分析表明, 得到的油状物中 $n(\mathrm{~K}): n(\mathrm{Ln}): n(\mathrm{P}): n(\mathrm{Ti})$ : $n(\mathrm{~W})$ 物质的量比为 $1: 1: 1: 2: 10$, 与化学式 $\mathrm{KLnH}_{3} \mathrm{PTi}_{2} \mathrm{~W}_{10} \mathrm{O}_{40}$ 一致, 化学式中需加三个质子以平衡 电荷. 众所周知, 多酸阴离子表面的端氧或桥氧易发生 质子化 ${ }^{[21-23]}$, 质子化的氢在水中比较难以电离成自由移 动的质子. 合成的离子液体水溶液的 $\mathrm{pH}>6$, 表明多酸 阴离子以 $\left[\mathrm{H}_{3} \mathrm{PTi}_{2} \mathrm{~W}_{10} \mathrm{O}_{40}{ }^{4-}\right]$ 形式存在, 其氢离子在水中 不发生电离.

\section{2 结构表征}

我们对合成的系列离子液体(离子液体 $1 \sim 8$ )、失水 后得到的固体(固体 $1 \mathrm{~s} \sim 8 \mathrm{~s}$ ) 以及加适量的水重新得到的 油状物(油状物 10 80) 进行了红外光谱(图 S2 S4) 和粉 末 $\mathrm{X}$-射线衍射测试(图 S5 S7), 确定离子液体 $\mathbf{1} \sim 8$ 、 固体 $1 \mathrm{~s} \sim 8 \mathrm{~s}$ 以及油状物 $10 \sim 80$ 分别具有相似的结构. 
含 $\mathrm{Ce}$ 的离子液体 2、固体 $\mathbf{2 s}$ 和油状物 20 的特征颜色 明显, 因此选择它们作为典例用于随后的讨论.

图 2 为 $\mathrm{K}_{7} \mathrm{PTi}_{2} \mathrm{~W}_{10} \mathrm{O}_{40}(\mathrm{a})$ 及合成的离子液体 $2(\mathrm{~b})$ 的 红外光谱, 可以看到它们的红外光谱类似, 没有因阳离 子不同而呈现明显差异. 在 1080 760 $\mathrm{cm}^{-1}$ 范围归属于 Keggin 型阴离子 $\mathrm{X}-\mathrm{O}_{\mathrm{a}} 、 \mathrm{M}=\mathrm{O}_{\mathrm{d}} 、 \mathrm{M}-\mathrm{O}_{\mathrm{b}} 、 \mathrm{M}-\mathrm{O}_{\mathrm{c}}$ 四个 键的特征振动峰清晰可见. 与完整的 Keggin 型(如: $\mathrm{PW}_{12} \mathrm{O}_{40}{ }^{3-}$ ) 阴离子比较, $1080 \mathrm{~cm}^{-1}$ 处振动峰䢃裂为 1081、1061、1042 $\mathrm{cm}^{-1}$ 处三个峰, 认为是两个 $\mathrm{Ti}$ 原子 取代 $\mathrm{W}\left(\mathrm{PW}_{12} \mathrm{O}_{40}{ }^{3-}\right.$ 中的两个 $\left.\mathrm{W}\right)$ 后阴离子对称性降低所 致. 这种由于结构对称性降低导致 IR 光谱峰发生䢃裂 的现象在以往的工作中也发现过 ${ }^{[23-24]}$.

研究发现, 水是该系列离子液体不可缺少的组分. 有趣的是系列离子液体经过干燥失水后成为透明固体. 固体 2s 的 IR 谱在 $1080 \mathrm{~cm}^{-1}$ 处归属于 $\mathrm{P}-\mathrm{O}_{\mathrm{a}}$ 键振动的 䢃裂峰变得不明显(图 2c). 失水后的固体 $2 \mathrm{~s}$ 加入适量水 中可以再得到油状物 2o, 其 IR 光谱在 $1080 \mathrm{~cm}^{-1}$ 处䢃 裂峰又清晰可见(图 2d). 这说明离子液体失水变成固体 后其多酸结构没有发生变化, 而且其结构对称性有所增 强. 同时, 水对于维持该系列离子液体结构稳定是必要 的. 类似的实验现象在其它离子液体体系也被报道 过 ${ }^{[19]}$.

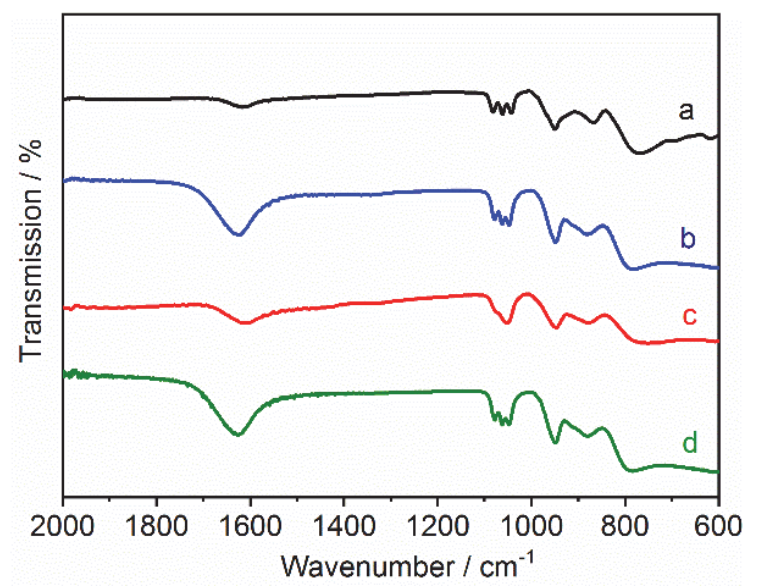

图 2 合成的 $\mathrm{K}_{7} \mathrm{PTi}_{2} \mathrm{~W}_{10} \mathrm{O}_{40}$ (a), 离子液体 2 (b), 固体 $2 \mathrm{~s}(\mathrm{c})$, 油状物 20 (d)的红外光谱图

Figure 2 IR spectra of (a) as-synthesized $\mathrm{K}_{7} \mathrm{PTi}_{2} \mathrm{~W}_{10} \mathrm{O}_{40}$, (b) ionic liquid 2, (c) solid 2s and (d) oil 20

通过 PXRD(图 3)考察了离子液体 2、固体 2s 以及 油状物 20 的结构变化, 并以 $\mathrm{K}_{7} \mathrm{PTi}_{2} \mathrm{~W}_{10} \mathrm{O}_{40}$ 作对比. 可 见, 在 $2 \theta=5^{\circ} \sim 50^{\circ}$ 范围内, $\mathrm{K}_{7} \mathrm{PTi}_{2} \mathrm{~W}_{10} \mathrm{O}_{40}$ 有明显的强衍 射峰, 表明其具有很好的结晶性. 而离子液体 2 、固体 2s 及油状物 20 的 PXRD 谱相似, 未见清晰的衍射峰, 表 明失(得)水过程中结构未发生变化, 仍为无定型体. 仔 细观察会发现, 固体 $2 \mathrm{~s}$ 的 PXRD 谱在低角度的宽衍射峰 略向高角度移动 $\left(2 \theta=8.2^{\circ} \rightarrow 8.7^{\circ}\right)$, 推测是失水引起的.

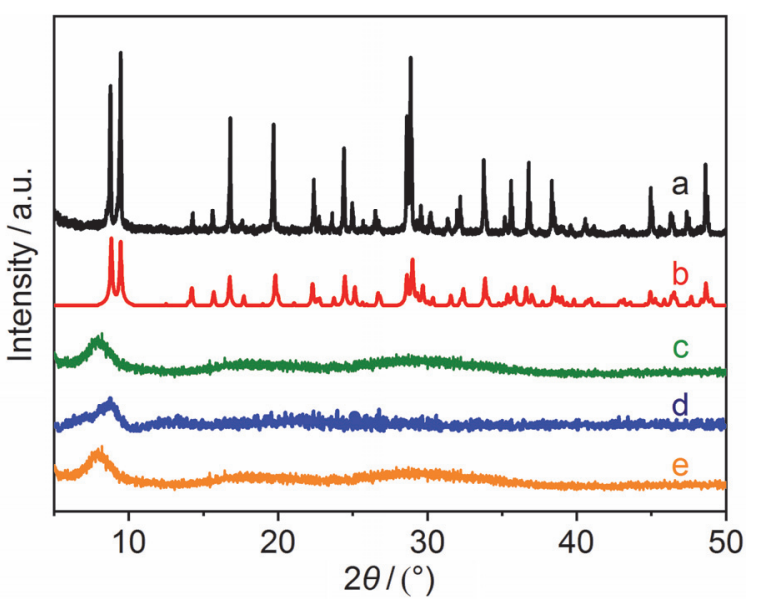

图 3 合成的 $\mathrm{K}_{7} \mathrm{PTi}_{2} \mathrm{~W}_{10} \mathrm{O}_{40}(\mathrm{a}), \mathrm{K}_{7} \mathrm{PTi}_{2} \mathrm{~W}_{10} \mathrm{O}_{40}$ 单晶模拟(b), 离子液体 2 (c), 固体 2s (d) 和油状物 $20(e)$ 的 PXRD 图

Figure 3 PXRD spectra of (a) as-synthesized $\mathrm{K}_{7} \mathrm{PTi}_{2} \mathrm{~W}_{10} \mathrm{O}_{40}$, (b) simulated $\mathrm{K}_{7} \mathrm{PTi}_{2} \mathrm{~W}_{10} \mathrm{O}_{40}$, (c) ionic liquid 2, (d) solid $2 \mathrm{~s}$ and (e) oil $2 \mathbf{o}$

\section{3 离子导电性质及电化学窗口}

电导率是研究离子液体导电性的重要物理参数. 在 $25{ }^{\circ} \mathrm{C}$ 时测得系列离子液体的电导率如表 1 所示, 它们 的离子电导率都在相同数量级, 均在 $10 \mathrm{mS} \cdot \mathrm{cm}^{-1}$ 以上.

表 1 系列离子液体 $\mathrm{KLnH}_{3} \mathrm{PTi}_{2} \mathrm{~W}_{10} \mathrm{O}_{40} \cdot x \mathrm{H}_{2} \mathrm{O}[\mathrm{Ln}=\mathrm{La}(\mathbf{1}), \mathrm{Ce}(\mathbf{2}), \operatorname{Pr}(\mathbf{3})$, $\mathrm{Nd}(\mathbf{4}), \operatorname{Sm}(\mathbf{5}), \operatorname{Eu}(\mathbf{6}), \operatorname{Er}(7), \mathrm{Yb}(\mathbf{8})]$ 的性质: 含水量, $x$ 数值, $25{ }^{\circ} \mathrm{C}$ 时电 导率, 电化学窗口

Table 1 Properties of the series of ionic liquids: water content, the value of $x$, conductivity at $25{ }^{\circ} \mathrm{C}$, electrochemical windows

\begin{tabular}{ccccc}
\hline Ionic liquid & $\mathrm{H}_{2} \mathrm{O}(w)$ & $x$ & $\sigma /\left({\left.\mathrm{mS} \bullet \mathrm{cm}^{-1}\right)}^{\mathrm{ECW} / \mathrm{V}}\right.$ \\
\hline $\mathbf{1}$ & 20.69 & 40.38 & 10.87 & 1.6 \\
$\mathbf{2}$ & 18.66 & 35.52 & 13.30 & 1.9 \\
$\mathbf{3}$ & 19.02 & 36.38 & 12.65 & 1.7 \\
$\mathbf{4}$ & 15.93 & 29.39 & 13.96 & 1.7 \\
$\mathbf{5}$ & 14.76 & 26.91 & 14.24 & 1.8 \\
$\mathbf{6}$ & 17.49 & 32.96 & 13.25 & 1.6 \\
$\mathbf{7}$ & 17.98 & 34.28 & 12.04 & 1.8 \\
$\mathbf{8}$ & 20.1 & 39.42 & 10.63 & 1.8 \\
\hline
\end{tabular}

研究了离子液体电导率随温度的变化关系, 发现系 列离子液体电导率均表现为在较低温度范围内随温度 升高而升高, 但在较高温度范围内随温度升高反而降 低. 以离子液体 2 为例, 从 25 到 $90{ }^{\circ} \mathrm{C}$ 每隔 $5{ }^{\circ} \mathrm{C}$ 测试其 电导率. 如图 4, 在 $25 \sim 65{ }^{\circ} \mathrm{C}$ 范围内, 电导率随温度升 高而增大 $\left(13.3 \sim 22.6 \mathrm{mS} \cdot \mathrm{cm}^{-1}\right)$, 而 $65{ }^{\circ} \mathrm{C}$ 以后, 其电导 率随温度升高显著降低, $90{ }^{\circ} \mathrm{C}$ 时电导率降至 1.22 $\mathrm{mS} \cdot \mathrm{cm}^{-1}$. 大量研究表明 ${ }^{[25-27]}$, 离子液体的电导率与其 粘度有关, 粘度增大, 离子迁移率降低, 电导率下降. 离子液体 2 在 $65 \sim 90{ }^{\circ} \mathrm{C}$ 温度范围内电导率快速下降, 认为是由于离子液体 2 组分中水逐渐失去, 导致其粘度 增大所致.

循环伏安法 $(\mathrm{CV})$ 是研究离子液体电化学窗口特性 的一种常用方法. 本工作采用常规三电极体系进行循环 伏安电化学测试, $\mathrm{Ag} / \mathrm{AgCl}$ 电极作参比电极. 图 5 所示为 
离子液体 2 在室温环境下的循环伏安曲线. 扫速为 20 $\mathrm{mV} \cdot \mathrm{s}^{-1}$ 时, 电势在 $-1.0 \sim-0.6 \mathrm{~V}$ 区间内存在一对可逆 的氧化还原峰, 还原峰归属于 $\mathrm{W}^{\mathrm{VI}}$ 还原为 $\mathrm{W}^{\mathrm{V}}$. 电势在 $-0.6 \sim 1.3 \mathrm{~V}$ 区间内无明显氧化还原峰, 电化学窗口为 $1.9 \mathrm{~V}$. 系列离子液体表现出相似的氧化还原行为(图 S8). 电化学窗口范围在 $1.6 \sim 1.9 \mathrm{~V}$.

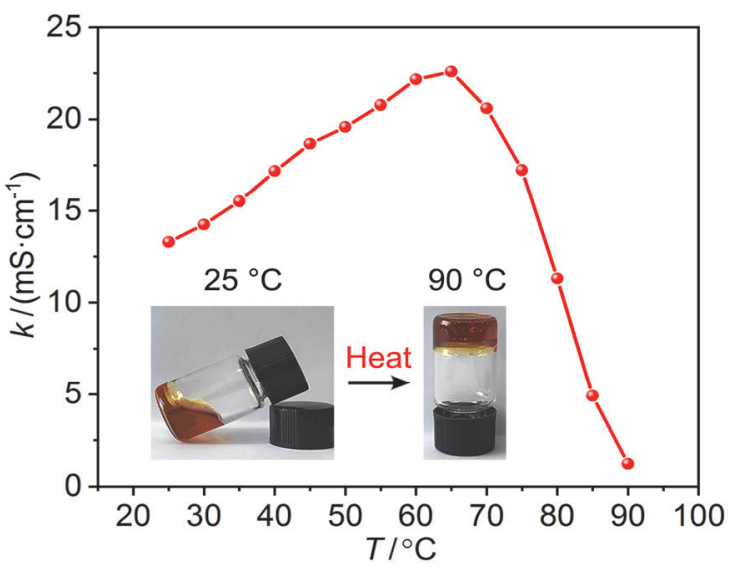

图 4 离子液体 $\mathbf{2}$ 的电导率随温度变化曲线. 插图: 离子液体 $\mathbf{2}$ 粘度 变化直观图

Figure 4 Conductivity of ionic liquid 2 at different temperatures. Inset: Visual representation of temperature-dependent viscosity change of the ionic liquid 2

以离子液体 2 为例研究扫速对循环伏安曲线的影响 (见图 5). 在 $20 \sim 100 \mathrm{mV} \cdot \mathrm{s}^{-1}$ 范围内改变扫速, $\mathrm{CV}$ 曲线 形状相似, 氧化峰和还原峰对称性很好, 表明其氧化还 原过程可逆. 同时可以看到, 还原峰电位随着扫速的增 加而略向负移, 而氧化峰略向正电位移动. 图 5 插图为 峰电流与扫速的变化关系. 氧化及还原峰电流与扫速 的平方根成正比, 表明该氧化还原过程为扩散控制过 程 ${ }^{[28]}$.

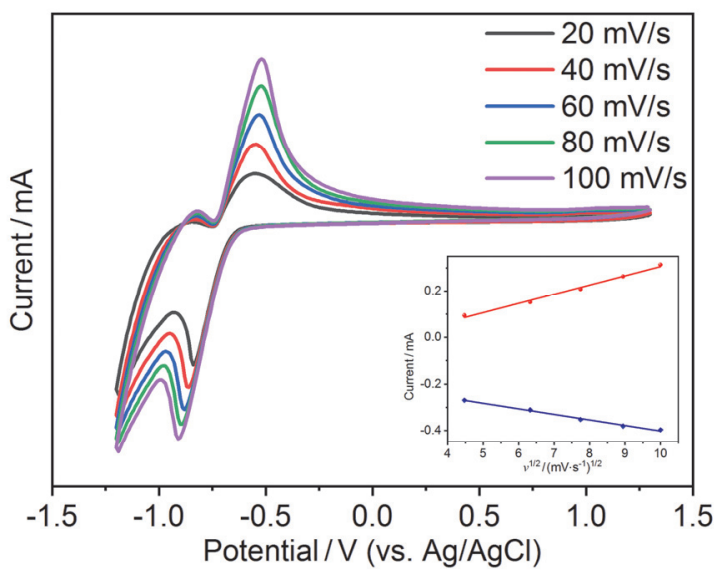

图 5 离子液体 $\mathbf{2}$ 的循环伏安曲线. 插图: 峰电流随扫速的变化关系 曲线

Figure 5 Cyclic voltammograms of ionic liquid 2. Inset: The dependence of cathodic peak and anodic peak currents on scan rates of ionic liquid 2

\section{4 离子液体与水的二元相行为}

我们发现, 在室温环境下该系列离子液体与水均不 互溶为两相, 离子液体相位于底部, 加热后混溶为均一 相. 我们通过浊点测试法研究了离子液体 2 和水的液液相平衡随温度变化关系. 图 6 所示为不同质量分数离 子液体 2 与水混合的相行为与温度的变化曲线. 可以看 到, 在离子液体 2 占组分质量分数低于 $50 \%$ 的测试范围 内, 随着离子液体 2 含量的增大, 由两相转变为均相的 温度逐渐升高. 而在离子液体 2 占组分质量分数高于 $50 \%$ 的测试范围内, 随着含量的增大其由两相转变为均 相的温度反而逐渐降低. 在高于 $85{ }^{\circ} \mathrm{C}$ 左右时, 离子液 体 2 任意含量的混合体系均为澄清均相.

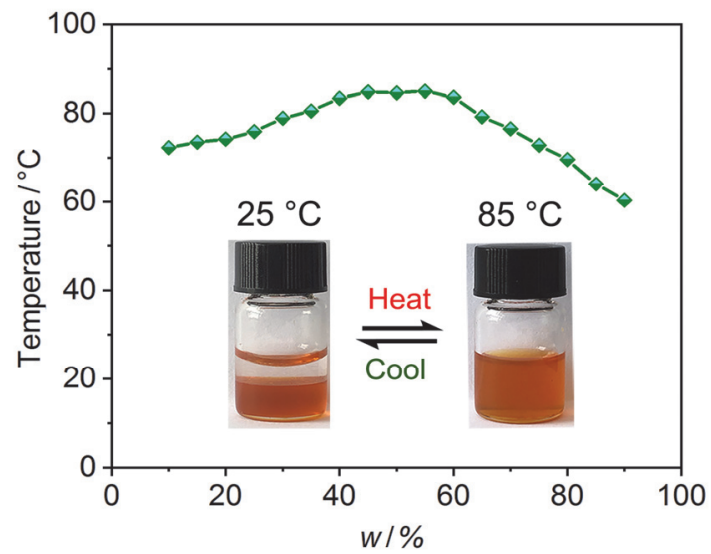

图 6 离子液体 2 和 $\mathrm{H}_{2} \mathrm{O}$ 液-液相平衡温度变化曲线. 插图: 温度响应 的离子液体 2 和 $\mathrm{H}_{2} \mathrm{O}$ (离子液体 $2, w=60 \%$ )动态相行为变化直观表示 图

Figure 6 Liquid-liquid equilibrium phase diagram of the ionic liquid 2/ $\mathrm{H}_{2} \mathrm{O}$ binary mixtures. Inset: Visual representation of temperaturedependent miscibility of the ionic liquid $\mathbf{2}$ with water (ionic liquid $\mathbf{2}, w=$ $60 \%)$

离子液体和溶剂(水或有机溶剂)的混溶性与温度变 化的相关性已被一些研究所发现 ${ }^{[29]}$, 一般分为两种情 况：(1)离子液体在溶剂中的溶解度随温度升高而增加, 在某一温度以上会形成均相, 这一相变过程称为上临界 溶解温度(upper critical solution temperature, UCST)相行 为. (2)离子液体在溶剂中的溶解度随温度升高而降低, 在高于某一温度时出现两相, 低于这一温度时为均相, 这种相变过程为下临界溶解温度(lower critical solution temperature, LCST)相行为. 本文报道的系列纯无机离子 液体在水中溶解度表现为随温度升高而明显增大，即 UCST 相行为. 这种随温度变化的动态相分离过程是非 常有趣的现象, 可以通过改变温度来建立离子液体/水 混合物的液-液相平衡. 基于这种特殊温度调节性质, 离子液体可应用于更多领域. 例如，萃取分离金属离子

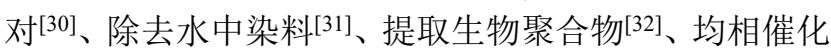
反应产物异相分离[32]等. 


\section{3 结论}

我们报道了系列纯无机离子液体, 它们由钛取代 Keggin 型铇磷酸阴离子和稀土离子及碱金属 $\mathrm{K}^{+}$构成. 在 $4 \sim 65{ }^{\circ} \mathrm{C}$ 范围表现为流动性很好的油状液体, 具有 较高的离子导电性(电导率均高于 $10 \mathrm{mS} \cdot \mathrm{cm}^{-1}$ ). 它们失 水会变成固体, 但失水后的固体放入适量水中可以重新 得到离子液体. 在室温环境下该系列离子液体和水混合 为两相, 加热到 $85{ }^{\circ} \mathrm{C}$ 左右时, 含 $\mathrm{Ce}$ 离子液体和水以任 意质量比混溶为均相. 该系列离子液体合成方法简单、 易操作、无污染. 这些特性将赋予该系列材料具有良好 的机械加工性及在催化和相分离等领域有潜在的应用.

\section{4 实验部分}

\section{1 试剂}

化学试剂均商业购买, 使用时未进一步纯化. 实验 中所用的水溶剂为蒸馏水.

\section{2 实验仪器和测试条件}

FT-IR 光谱使用 Alb Centauri FTIR 分光光度计在 $400 \sim 4000 \mathrm{~cm}^{-1}$ 范围内记录. 热重分析使用 PerkinElmer $\mathrm{TGA}$ 仪器在 $\mathrm{N}_{2}$ 气氛中以 $10{ }^{\circ} \mathrm{C} \cdot \mathrm{min}^{-1}$ 的速率从室温加热 到 $600{ }^{\circ} \mathrm{C}$ 测得. 用 Rigaku D/MAX-3 仪器在 $293 \mathrm{~K}$ 下 $\mathrm{CuK} \alpha$ 辐射 $2 \theta=5^{\circ} \sim 50^{\circ}$ 范围内进行 PXRD 测试. 元素分 析通过 PLASMA-SPEC (I)电感耦合等离子体原子发射 光谱(ICP)测得. 循环伏安曲线 $(\mathrm{CV})$ 使用 CHI760E 电化 学工作站记录. 电导率使用 DDS-307 电导率仪测得.

\section{3 液一液相平衡测试}

离子液体 2 /水的液-液相平衡随温度变化曲线通过 改进文献 ${ }^{[31]}$ 方法测得. 将装有不同质量分数的离子液 体 2 和水的玻璃瓶浸入配有温度计的恒温水浴锅中, 加 热搅拌至呈均相, 再逐渐冷却, 记录第一时间出现浑浊 迹象的温度即为浊点温度, 并作为液-液相转变的温度, 重复 3 次测试. 注意: 在第一次测试初步观察到浊点温 度范围时, 后两次测试在接近浊点温度时尽量控制温度 变化速率, 以不超过 $10{ }^{\circ} \mathrm{C} \cdot \mathrm{h}^{-1}$ 为宜.

\section{References}

[1] Hallett, J. P.; Welton, T. Chem. Rev. 2011, 111, 3508.

[2] Lei, Z. G.; Chen, B. H.; Koo, Y. M.; MacFarlane, D. R. Chem. Rev. 2017, 117, 6633 .

[3] Rogers, R. D.; Seddon., K. R. Science 2003, 302, 792.

[4] Kang, X. C.; Sun, X. F.; Han, B. X. Adv. Mater. 2016, 28, 1011.
[5] Li, S. N.; Zhao, W. X.; Liu, Y. J.; Liu, Z. Q.; Ying, A. G. Chin. J. Org. Chem. 2020, 40, 1835 (in Chinese). (李胜男, 赵雯辛, 刘玉 静, 刘中秋, 应安国, 有机化学, 2020, 40, 1835.)

[6] Kore, R.; Berton, P.; Kelley, S. P.; Aduri, P.; Katti, S. S.; Rogers, R. D. ACS Catal. 2017, 7, 7014.

[7] Zhou, Z. H.; Chen, K. H.; He, L. N. Chin. J. Chem. 2019, 37, 1223.

[8] Yao, W. H.; Wang, H. Y.; Pei, Y. C.; Chen, Y. H.; Li, Z. Y.; Wang, J. Y. RSC Adv. 2017, 7, 11297.

[9] Sun, T. X.; Shen, X. H.; Chen, Q. D. Acta Phys.-Chim. Sin. 2015, 31,32 (in Chinese). (孙涛祥, 沈兴海, 陈庆德, 物理化学学报, 2015, 31, 32).

[10] Bara, J. E.; Camper, D. E.; Gin, D. L.; Noble, R. D. Acc. Chem. Res. 2010, 43, 152.

[11] Xing, H. B.; Liao, C.; Yang, Q. W.; Veith, G. M.; Kun, G. B.; Sun, X. G.; Ren, Q. L.; Hu, Y. S.; Dai, S. Angew. Chem. Int. Ed. 2014, 53, 2099.

[12] Kang, S. S.; Fan, S. C.; Liu, Y.; Wei, Y. C.; Li, Y.; Fang, J. G.; Meng, C. Z. Acta Chim. Sinica 2019, 77, 647 (in Chinese). (康树森，范少 聪, 刘岩, 魏彦存, 李营, 房金刚, 孟垂舟, 化学学报, 2019, 77, 647).

[13] Miras, H. N.; Yan, J.; Long, D. L.; Cronin, L. Chem. Soc. Rev. 2012, 41,7403 .

[14] Wang, S. S.; Yang, G. Y. Chem. Rev. 2015, 115, 4893.

[15] Wei, Z. Y.; Chang, Y. L.; Yu, H.; Han, S.; Wei, Y. G. Acta Chim. Sinica 2020, 78, 725 (in Chinese). (魏哲宇, 常亚林, 余焓, 韩生, 魏永革, 化学学报, 2020, 78, 725).

[16] Leng, Y.; Wang, J.; Zhu, D. R.; Ren, X. Q.; Ge, H. Q.; Shen, L. Angew. Chem. Int. Ed. 2009, 48, 168.

[17] Herrmann, S.; Kostrzewa, M.; Wierschem, A.; Streb, C. Angew. Chem. Int. Ed. 2014, 53, 13596.

[18] Qiao, Y. X.; Hou, Z. S.; Li, H.; Hu, Y.; Feng, B.; Wang, X. R.; Hua, L.; Huang, Q. F. Green Chem. 2009, 11, 1955.

[19] Dai, L. Y.; Yu, S. Y.; Shan, Y. K.; He, M. Y. Eur. J. Inorg. Chem. 2004, 237.

[20] Domaille, P. J.; Knoth, W. H. Inorg. Chem. 1983, $22,818$.

[21] Bi, L. H.; Hussain, F.; Kortz, U.; Sadakane, M.; Dickman, M. H. Chem. Commun. 2004, 1420.

[22] Guan, W.; Yan, L. K.; Su, Z. M.; Liu, S. X.; Zhang, M.; Wang, X. H. Inorg. Chem. 2005, 44, 100.

[23] Wang, X. N.; Liu, S. X.; Li, S. J.; Xie, R. H.; Zhang, X.; Liu, Y. W. Chem. J. Chinese Universities. 2013, 34, 1047 (in Chinese). (王雪 娜, 刘术侠, 李书军, 谢瑞红, 张金金, 刘艺伟, 高等学校化学学 报, 2013, 34, 1047).

[24] Wang, C. L.; Liu, S. X.; Xie, L. H.; Ren, Y. H.; Liang, D. D.; Sun, C. Y.; Cheng, H. Y. Polyhedron 2007, 26, 3017.

[25] Ichikawa, T.; Yoshio, M.; Hamasaki, A.; Taguchi, S.; Liu, F.; Zeng, X.-B.; Ungar, G.; Ohno, H.; Kato, T. J. Am. Chem. Soc. 2012, 134, 2634.

[26] Wu, X. F.; Zhou, X. H.; Wu, Q. Y.; Yan, W. F. New J. Chem. 2016, 40, 7923.

[27] Zheng, Q. G.; Liu, H.; Xia, Q.; Liu, Q. S.; Mou, L. Acta Phys.-Chim. Sin. 2017, 33, 736 (in Chinese). (郑其格, 刘惠, 夏泉, 刘青山, 牟 林, 物理化学学报, 2017, 33, 736.)

[28] Wang, C.; Ying, J.; Mou, H. C.; Tian, A. X.; Wang, X. L. Inorg. Chem. Front. 2020, 7, 3882.

[29] Qiao, Y. X.; Ma, W. B.; Theyssen, N.; Chen, C.; Hou, Z. S. Chem. Rev. 2017, 117, 6881.

[30] Depuydt, D.; Van den Bossche, A.; Dehaen, W.; Binnemans, K. Chem. Commun. 2017, 53, 5271.

[31] Blesic, M.; Gunaratne, H. Q. N.; Jacquemin, J.; Nockemann, P.; Olejarz, S.; Seddon, K. R.; Strauss, C. R. Green Chem. 2014, 16, 4115.

[32] Fukaya, Y.; Ohno, H. Phys. Chem. Chem. Phys. 2013, 15, 4066.

(Cheng, B.) 